\title{
Optimal Radio Resource Management in Multihop Relay Networks
}

\author{
Tolga Girici \\ Dept. of Electrical and Electronics Eng. \\ TOBB Economics and Technology University \\ Ankara,06560, Turkey \\ tgirici@etu.edu.tr
}

\author{
Chenxi Zhu, Jonathan Agre \\ Fujitsu Labs of America \\ 8400 Baltimore Ave., Suite 302 \\ College Park, MD 20740 \\ \{chenxi.zhu,j.agre\}@ fla.fujistsu.com
}

\author{
Anthony Ephremides \\ Institute for Systems Research \\ University of Maryland \\ College Park, Maryland 20740 \\ etony@umd.edu
}

\begin{abstract}
Multihop relay networks (MR) use relay stations (RS) to extend or enhance the coverage of a base station (BS) in a cellular network. The base station is attached to a wired backhaul. Relay stations use wireless transmission to connect to the base station and to the mobile stations (MS), while direct BS-MS connection is also possible. Low cost relay technologies are recently proposed for OFDM based broadband wireless technologies. Especially for low-cost relay stations that have single radio interface how to share the channels and allocate proper amount of bandwidth/power to the base station (BS), relay stations (RSs) and the mobile stations (MSs) is an important issue. This work develops methods for radio resource management in MR networks supporting heterogeneous traffic. We propose a scheduling method, where the transmission of base station to each relay station and transmissions of each relay station to its respective mobile stations (i.e. composite links) are scheduled in a TDMA fashion and the resource allocation in those microcells are performed in an OFDMA manner. We numerically evaluate the performance of our proposed scheme with respect to a number of parameters.
\end{abstract}

\section{INTRODUCTION}

Broadband wireless networks are designed to be able to provide mobile high rate and heterogenous services that have various quality of service $(\mathrm{QoS})$ requirements. In recent years several broadband air interface technologies have been developed to provide Internet access multimedia services to end users. The most notable of these technologies is mobile WiMax. Based on the recently developed IEEE 802.16e standard [1], [2], WiMax is a cellular network, where a Base Station (BS) connects mobile stations (MS) to various networks linked to the BS. Transmission in WiMax networks is based on OFDMA, where several modulation, coding and power allocation schemes are allowed to give more degrees of freedom to resource allocation [3], [4]. WiMax networks are envisaged to provide service to a cellular area of size up to 50 miles. In such a network, users in different parts of a cell usually experience different signal qualities and thus different degrees of QoS. This is because the channel quality of a user depends on many factors including path loss, shadowing and interference. Users at the cell edge often suffer from bad channel conditions and observe lower SINR. In an urban environment, big buildings pose a serious blockage to users behind and sometimes generate coverage holes. Signal penetration and attenuation inside buildings or tunnels also degrade the signal quality significantly. Often it is not possible to improve the signal qualities to these under-serviced areas by increasing the transmission power or changing the antenna configurations. Reducing the cell size and deploying more base stations will improve the situation, but this is often not possible due to limited access to traditional cell sites and wired backhaul links and the associated high operating cost. Using radio relay stations is an effective way to increase the signal quality of the users by replacing a long, low quality link between a Base Station(BS) and a Mobile Station(MS) with multiple shorter, high quality links through one or multiple Relay Stations (RS). As RSs do not require their own wired backhauls, and are often less sophisticated than a full functional BS, RSs are less expensive to deploy and operate than a traditional base station. The standard for relay in WiMax networks is being developed by the $802.16 j$ Relay working group [5].

Use of relay in cellular systems were studied in [6], [7], [8] and [9]. Most of these works considered solely data traffic. The aim in [6], [7] was improving the aggregate throughput or spectral efficiency by using relays in broadband systems, while in [8] fair subchannel allocation algorithms were proposed and in [9] a heuristic was proposed to jointly improve outage probability and throughput. Unlike all these works we distinguish different traffic types and propose a frame-by-frame scheduler, where in each time slot, the time slots, subcarriers and power in a frame are jointly allocated to each transmission. Our algorithm provides proportional fairness for data users, while satisfying delay requirements for real time sessions. Proportional fairness is a good balance between throughput and fairness for data users. We also convert delay requirements of real time users into rate requirements and treat them as constraints in the optimization problem as in our previously proposed resource allocation scheme for a system without relay stations in [10].

In a typical multihop relay network the traffic between the BS and MSs can be forwarded via multiple hops through RSs. However in this work we assume that there is at most one RS between the BS and a MS. A RS communicates to the BS like a MS, and communicates with the MS in its coverage area (called RS-microcell) like a BS. Cost of RSs can be reduced by allowing a single transceiver at each RS. Therefore a RS cannot transmit and receive simultaneously, which is a major characteristic of our system model. We describe the system model in Section II in detail. In line with recent IEEE 802.16j 
standard the BS schedules microcell transmissions in a TDMA manner in a MMR frame. The BS first allocates a time interval (subframe) of the frame to each microcell, as explained in Section III. In the second stage of allocation the BS treats each microcell separately; it optimally divides the subframe for BS-RS and RS-MS composite links and also allocates the available bandwidth and power to each link in the microcell, which is explained in Section IV. We numerically evaluate the proposed algorithm in Section VI and compare its performance with that of the algorithm previously proposed for a network of single BS in [10].

\section{System Model AND Notation}

Figure 1 shows a typical multihop relay (MR) network. The base station is at the center, and there are a number of RSs located in the cell area. We assume that the MSs are located randomly in the cell are and they are fixed. Relay stations are also fixed and each MS is assigned to the BS or one of the RSs, based on the distance. ${ }^{1}$

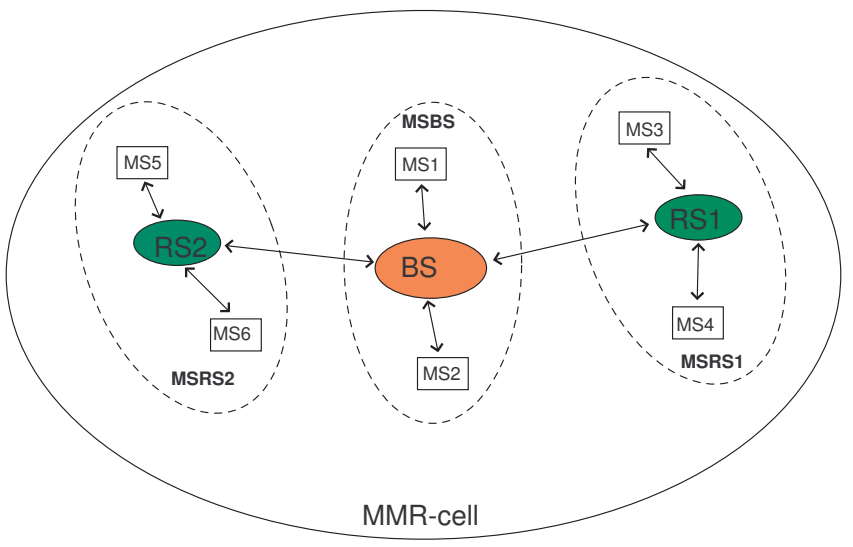

Fig. 1. Topology of a MR cell with a BS and two relay stations $\left(R S_{1}\right.$ and $\left.R S_{2}\right)$. The BS is serving the MSs in the set $M S_{B S}$ directly $\left(M S_{1}\right.$ and $\left.M S_{2}\right)$. Two relay stations $\left(R S_{1}, R S_{2}\right)$ are used to extend the coverage of BS and serve MSs in the set $M S_{R S 1}\left(M S_{3}, M S_{4}\right)$ and $M S_{R S 2}\left(M S_{5}, M S_{6}\right)$. The MR cell includes the coverage area of the BS and all the RSs.

In this work we consider frame by frame downlink resource allocation. Total frame duration is $T_{f}$ seconds and it is divided into time slots of duration $T_{s}$. Total bandwidth is $W \mathrm{~Hz}$, which is divided into $N_{s u b}$ subchannels of $W_{s u b} \mathrm{~Hz}$ bandwidth. We assume PUSC as the subchannelization method [1], where a subchannel is formed by randomly sampling subcarriers from the entire frequency range. Because of sampling, all subchannels are of equal channel quality with respect to a user. While modeling the allocation problem we will consider time and bandwidth as a continuously divisible quantity. After finding the optimal values, we will quantize them to the integer multiples of subchannel bandwidth and time slot duration. We assume for simplicity that each user demands only one type of traffic, data, video streaming or voice. Let $U_{D}$ and $U_{R}$ be

\footnotetext{
${ }^{1}$ We consider a fixed system but simulate mobility of MSs through fast Rayleigh fading and slow Log-normal shadowing.
}

the set of data and real time sessions. Set of nodes assigned to $R S_{i}$ is denoted as $M S_{R S i}$ and set of nodes directly connected to $\mathrm{BS}$ is called $M S_{B S}$. This assignment is based on path loss. A node is assigned directly to the BS or one of the RSs that maximizes the received signal strength. We assume that this assignment is fixed. The BS keeps separate queues for each user, while each RS also keeps separate queues for the set of nodes $M S_{R S}$. We make the following definitions:

- Microcell: A microcell is formed by a group of MSs directly connected to a station (BS or RS). Let M-1 be the number of RSs. Including the MSs directly connected to the $\mathrm{BS}$, there are $\mathrm{M}$ microcells. Let $M C_{i}$ be the $i^{t h}$ microcell, where $M C_{M}$ denotes the microcell that contains the MSs directly connected to the BS. In the example in Figure 1 there are 3 microcells.

- Composite Link: There are three types of composite links. The set of transmissions through $B S \rightarrow R S_{i}, R S_{i} \rightarrow M S_{R S i}$ for all $i=1, \ldots, M-1$ and $B S \rightarrow M S_{B S}$ are all composite links. Figure 2 illustrates a typical downlink frame. As seen in the figure, transmissions belonging to different composite links are scheduled in a TDMA fashion in a downlink frame. As an example in Figure 1, there are 5 composite links and hence the downlink frame is divided into 5 TDMA subframes.

- Tandem queue: A tandem queue $l_{j}$ is the two cascading queues $B S \rightarrow R S_{i} \rightarrow M S_{j}$, where $j \in M S_{R S i}$. Let $h_{j}^{B S}$ and $h_{j}^{R S}$ be the channel gains for the links $B S \rightarrow R S_{i}$ and $R S_{i} \rightarrow M S_{j}$, respectively. Obviously $h_{j}^{B S}=h_{k}^{B S}$ for all $j, k \in M S_{R S_{i}}$, because those queues follows the same $B S \rightarrow R S_{i}$ link. Let $q_{j}^{B S}$ and $q_{j}^{R S}$ be the number of bits waiting in those queues to be transmitted.

In an MR network, bandwidth is often limited and has to be shared by the base stations and multiple relay stations to serve all the MSs in the MR-cell. We assume that a relay station has a single radio interface in order to reduce the cost, which also mandates the RS to use the same channel to communicate with the BS and with its MSs (and potentially with other RSs). Because of the single interface constraint of relay stations, transmissions $B S \rightarrow R S_{i}$ and $R S_{i} \rightarrow M S_{R S i}$ should also be scheduled in a TDMA fashion. Considering this and for simplicity we follow a TDMA approach in scheduling transmissions of each composite link.

Let $P^{B S}$ and $P^{R S}$ be the available power budget for the $\mathrm{BS}$ and each RS, respectively. We consider a channel with Rayleigh fading and Log-normal shadowing. At each time frame the channel gain is assumed constant and we consider an equivalent AWGN channel. In order to determine the bandwidth efficiency as a function of SNR, we use the values in IEEE 802.16 standard [5]. A number of modulation/coding pairs that correspond to SINR thresholds and spectral efficiency values in [5]. Considering the SINR-spectral efficiency relation and by using $\beta=0.25$, it is reasonable to use the following function for the number of bits that is transmitted through a link 


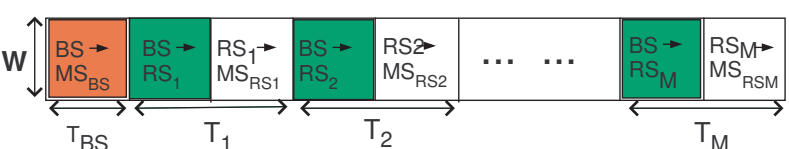

(a)

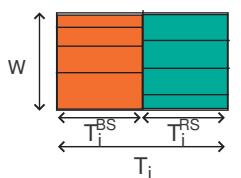

(b)

Fig. 2. (a) Downlink subframe for the TDD frame structure of a MMR cell. BS and M RSs share the DL subframe on a TDMA basis. On the downlink, $T_{i}$ includes all the time slots assigned to the traffic $B S \rightarrow R S i \rightarrow M S_{R S i}$. (b) shows a microcell subframe. Transmissions in $B S \rightarrow R S i$ and $R S i \rightarrow M S_{R S i}$ composite links are given $T_{i}^{B S} T_{i}^{R S}$ seconds.

$$
r_{j}^{\phi}=T_{i}^{\phi} w_{j}^{\phi} \log \left(1+\frac{\beta p_{j}^{\phi} h_{j}^{\phi}}{w_{j}^{\phi} N_{0}}\right), \phi=B S, R S, j \in M S_{R S i}
$$

Here $T_{i}^{\phi}$ is the part of the frame (in seconds) that is allocated to the composite link $\phi$ (BS or RS) of microcell $i$. Let $p_{j}^{\phi}$ $w_{j}^{\phi}$ be the power and bandwidth user $\mathrm{j}$ in microcell $i$ gets $(\phi=B S(R S)$ for the BS-RS (RS-MS) link). In order to decrease the dimensionality of the problem we divide it into two parts as follows:

1) Cellular Time Allocation: In this step we consider a TDMA scheme among composite links, where all sessions in a composite link transmit simultaneously in a $T_{i}^{\phi}$ second subframe and share the available bandwidth and power. Before performing TDMA allocation we also determine rate requirements for each real time session.

2) Microcell Resource allocation: In this step we separately perform joint power/bandwidth allocation for each $B S \rightarrow$ $R S \rightarrow M S_{R S i}, \forall i \in M C$.

\section{Cellular Time Allocation}

In this section we consider resource allocation in a single microcell, which includes the transmissions through composite links $B S \rightarrow R S_{i}$ and $R S_{i} \rightarrow M S_{R S i}, i \in M C$. For the data sessions let $R_{j}^{B S}$ and $R_{j}^{R S}$ be the time-averaged transmission rate through the access and relay links of tandem queue of data user $j \in$ $M S_{R S i} \cap U_{D}$. For the real time sessions, let $r_{j}^{c, B S}$ and $r_{j}^{c, R S}$ be the required rates for the tandem queues of session $j$ at the current frame.

\section{A. Real Time Session Rates}

First a number of real time session links are chosen in BSRS and RS-MS composite links to be transmitted in the current frame. We use the following user satisfaction value for real time sessions:

$$
U S V_{j}^{\phi}(t)=-\frac{\log \left(\delta_{j}\right)}{D_{j}^{\max }} D_{j}^{\phi} \log \left(1+\frac{\beta P^{\phi} h_{j}^{\phi}(t)}{N_{0} W}\right) \frac{\lambda_{j}}{R_{j}^{\phi}(t)}
$$

This metric resembles the Largest Weighted Delay First (LWDF) metric except the $\lambda_{j} / R_{j}^{\phi}(t)$ term at the end. Here $\lambda_{j}$ is the bit arrival rate and $R_{j}^{\phi}(t)$ is the service rate for user $\mathrm{j}$, where $\phi=B S$ for the BS-RS transmission and $\phi=R S$ for RS-MS transmission. Service rate is updated as $R_{j}^{\phi}(t+1)=\alpha R_{j}^{\phi}(t)+$ $(1-\alpha) r_{j}^{\alpha}(t) . D_{j}^{\max }$ and $D_{j}^{\phi}$ are the maximum allowable delay and current head of line delay for the link. Parameter $\delta_{j}$ is typically chosen as 0.05 and reflects the probability of exceeding the delay constraint. The BS chooses a number of real time sessions according to this metric, where $U_{R}^{\prime}$ denotes the set of chosen real time users. The rate constraint for a chosen real time session is defined as:

$$
r_{j}^{c, \phi}\left(q_{j}^{\phi}(t), \omega_{j}^{\phi}(t)\right)=\max \left(\lambda_{j}, \frac{q_{j}^{\phi}(t)}{D_{j}^{\max } 0.5 \omega_{j}^{\phi}(t)}\right), j \in U_{R}^{\prime}
$$

Here $\omega_{j}^{\phi}$ is the transmission frequency of the corresponding link of user $\mathrm{j}$, which is calculated as follows:

$$
\omega_{i}(t)=\alpha \omega_{j}^{\phi}(t-1)+(1-\alpha) I\left(r_{j}^{\phi}(t)>0\right),
$$

where $I\left(r_{j}^{\phi}(t)>0\right)$ is the function that takes value one if the node receives packets in time slot $t$, zero otherwise. Therefore this frequency decreases if the link transmits less and less frequently. Using this frequency expression in the rate function, we compensate for the lack of transmission in the previous time slots possibly due to bad channel conditions.

\section{B. Time Allocation for each Microcell}

In this section we will propose a method for allocating time intervals for each microcell. In order to simplify this process we assume uniform power allocation to each subchannel. In this case let the spectral efficiency at each link be defined as, $S_{j}^{\phi}=\log \left(1+\frac{P^{\phi}}{n_{j}^{\phi} W}\right), \forall j \in U, \phi=B S, R S$. Multiplying this by time and bandwidth we obtain the number of nats transmitted as $r_{j}^{\phi}=T_{i}^{\phi} w_{j}^{\phi} S_{j}^{\phi}$ nats. We can define time-bandwidth product as $b_{j}^{\phi}=T_{i}^{\phi} w_{j}^{\phi}$ for $j \in M S_{R S i}$ and allocate resources subject to a time bandwidth constraint $\sum_{j \in U_{D} \cup U_{R}} b_{j}^{B S}+b_{j}^{R S} \leq W T_{f}$. For a real time session link $\mathrm{j}$ required time bandwidth product can be directly computed as $b_{j}^{\phi}=\frac{r_{j}^{c, \phi}}{S_{j}^{\phi}}, \phi=B S, R S$. So we can do a resource allocation only for data sessions and subject to the constraint $\sum_{j \in U_{D}} b_{j}^{B S}+b_{j}^{R S} \leq\left(W T_{f}\right)^{\prime}=W T_{f}-$ $\sum_{j \in U_{R}} r_{j}^{c, B S} / S_{j}^{B S}+r_{j}^{c, R S} / S_{j}^{R S}$. We formulate a proportional fair time-bandwidth allocation problem for data users as follows:

$$
\max _{\mathbf{b}, \mathbf{r}} \sum_{j \in U_{D}} \log \left(\alpha_{j} R_{j}+\left(1-\alpha_{j}\right) r_{j}\right)
$$




$$
\begin{aligned}
\sum_{j \in U_{D}} b_{j}^{B S}+b_{j}^{R S} & \leq\left(W T_{f}\right)^{\prime} \\
b_{j}^{\phi} S_{j}^{\phi} & \geq r_{j}, \phi=B S, R S, \forall j U_{D}
\end{aligned}
$$

where $R_{j}=\min \left\{R_{j}^{B S}, R_{j}^{R S}\right\}$ be the average received rate through the tandem queue. The problem above has a concave objective function increasing in each data session rate. The constraint set is convex, hence we can solve the problem by using Lagrange multipliers.

$$
\begin{gathered}
L\left(\mathbf{b}, \mathbf{r}, \lambda_{b}, \overline{\lambda_{r}}\right)=\sum_{j \in U_{D}} \log \left(\alpha_{j} R_{j}+\left(1-\alpha_{j}\right) r_{j}\right)+\lambda_{b}\left(\left(W T_{f}\right)^{\prime}\right. \\
\left.-\sum_{j \in U_{D}} b_{j}^{B S}+b_{j}^{R S}\right)+\sum_{\phi=B S, R S} \sum_{j \in U_{D}} \lambda_{r}^{j, \phi}\left(b_{j}^{\phi} S_{j}^{\phi}-r_{j}\right)
\end{gathered}
$$

By using standard techniques we find that solution of this problem requires a simple binary search on $\lambda_{b}$ that solves the following equation:

$$
S_{b}\left(\lambda_{b}\right)=W T_{f}
$$

where,

$$
\begin{gathered}
r_{j}\left(\lambda_{b}\right)=\left[\frac{1}{\lambda_{b}\left(\frac{1}{S_{j}^{B S}}+\frac{1}{S_{j}^{R S}}\right)}-\widetilde{\alpha} R_{j}\right]^{+} \forall j \in U_{D} \\
S_{b}\left(\lambda_{b}\right)=\sum_{j \in U_{D}} r_{j}\left(\lambda_{b}\right)\left(\frac{1}{S_{j}^{B S}}+\frac{1}{S_{j}^{R S}}\right)+\sum_{j \in U_{R}}\left(\frac{r_{j}^{c, B S}}{S_{j}^{B S}}+\frac{r_{j}^{c, R S}}{S_{j}^{R S}}\right)
\end{gathered}
$$

As we see sum of time-bandwidth resources is a monotonic decreasing function of $\lambda_{b}$. Based on these result of this optimization in order to share the frame in a TDMA manner time allocated to composite links in microcell $i$ can be computed as

$$
T_{i}^{\phi}\left(\lambda_{b}\right)=\frac{1}{W} \sum_{j \in M S_{R S i}} r_{j}\left(\Lambda_{b}\right) b_{j}^{\phi}, \phi=B S, R S, \forall i \in M C
$$

\section{Feasibility of the Problem}

The solution above is requires a feasibility condition. By feasibility we mean that the available resources are enough to support at least the required rates for real time sessions. Let us define $\underline{T}_{i}^{B S}$ and $\underline{T}_{i}^{R S}$ be the minimum required time to support the real time sessions in BS-RS and RS-MS links. We can find them by taking the limit

$$
\underline{T}_{i}^{\phi}=\lim _{\lambda_{b} \rightarrow \infty} T_{i}^{\phi}\left(\lambda_{b}\right)
$$

Looking at the rate equation in (30) we see that limit $\lambda_{b} \rightarrow$ $\infty$ makes the data session rates equal to zero and real time sessions are unaffected. If $\sum_{i \in M C} \underline{T}_{i}^{B S}+\underline{T}_{i}^{R S}>T_{f}$, then we find the non-zero-rate link with worst channel condition and change its required rate equal to zero.

\section{Composite Link Resource Allocation}

In this section we consider resource allocation in a single microcell. Let us consider transmission through composite links $B S \rightarrow R S_{i}$ and $R S_{i} \rightarrow M S_{R S i}$. Let $T_{i}$ be the time allocated to microcell $i$. At this stage the BS makes the power $(\mathbf{p}=$ $\left.\left\{p_{j}^{B S}, p_{j}^{R S} \mid j \in M S_{R S i}\right\}\right)$, bandwidth $\left(\mathbf{p}=\left\{w_{j}^{B S}, w_{j}^{R S} \mid j \in M S_{R S i}\right\}\right)$ for links in this microcell and time $\left(\mathbf{T}_{i}=\left\{T_{i}^{B S}, T_{i}^{R S}\right\}\right)$ allocation for the $B S-R S_{i}$ and $R S_{i}-M S_{R S i}$ composite links (illustrated in Figure 2(b)). The objective is to maximize the log sum of data rates for each tandem queue supporting data sessions. The constraints are the real time sessions rate requirements defined in the previous part, and total power, rate, time constraints. The constrained optimization problem is formulated as follows:

$$
\begin{aligned}
& \max _{\mathbf{w}, \mathbf{p}, \mathbf{T}_{i}} \sum_{j \in M S_{R S i} \cap U_{D}} \log \left(\alpha R_{j}+(1-\alpha) r_{j}\right) \\
& \text { s.t. } T_{i}^{B S}+T_{i}^{R S} \leq T_{i} \\
& \sum_{j \in M S_{R S i}} p_{j}^{\phi} \leq P^{\phi} \\
& \sum_{j \in M S_{R S i}} w_{j}^{\phi} \leq W \\
& T_{i}^{\phi} w_{j}^{\phi} \log \left(1+\frac{p_{j}^{\phi}}{n_{j}^{\phi} w_{j}^{\phi}}\right) \\
& T_{i}^{\phi} w_{j}^{\phi} \log \left(1+\frac{p_{j}^{\phi}}{n_{j}^{\phi} w_{j}^{\phi}}\right) \geq r_{j}, \forall j \in M S_{R S i} \cap U_{D}
\end{aligned}
$$

where, $\phi=B S, R S$ and $n_{j}^{\phi}=N_{0} /\left(h_{j}^{\phi} \beta\right)$. The problem above has a concave objective function increasing in each data session rate. The constraint set is convex, hence we can solve the problem by using Lagrange multipliers.

$$
\begin{gathered}
L\left(\mathbf{w}, \mathbf{p}, \mathbf{T}_{i}, \overline{\lambda_{p}}, \overline{\lambda_{w}}, \overline{\lambda_{r}}, \lambda_{T}\right)=C_{i}\left(\mathbf{w}, \mathbf{p}, \mathbf{T}_{i}\right)+\lambda_{T}\left(T_{i}-T_{i}^{B S}-T_{i}^{R S}\right) \\
+\sum_{\phi=B S, R S} \lambda_{p}^{\phi}\left(P^{\phi}-\sum_{j \in M S_{R S i}} p_{j}^{\phi}\right)+\sum_{\phi=B S, R S} \lambda_{w}^{\phi}\left(W-\sum_{j \in M S_{R S i}} w_{j}^{\phi}\right) \\
+\sum_{\phi=B S, R S} \sum_{j \in M S_{R S i} \cap U_{D}} \lambda_{j}^{r, \phi}\left(T_{i}^{\phi} w_{j}^{\phi} \log \left(1+\frac{p_{j}^{\phi}}{n_{j}^{\phi} w_{j}^{\phi}}\right)-r_{j}\right) \\
+\sum_{\phi=B S, R S} \sum_{j \in M S_{R S i} \cap U_{R}} \lambda_{j}^{r, \phi}\left(T_{i}^{\phi} w_{j}^{\phi} \log \left(1+\frac{p_{j}^{\phi}}{n_{j}^{\phi} w_{j}^{\phi}}\right)-r_{j}^{0, \phi}\right)
\end{gathered}
$$

The problem can be solved by taking derivative with respect to resources and Lagrange multipliers. Since the rate is an increasing function of resources the optimal can be achieved only when all the time, power and bandwidth is used. Therefore all Lagrange multipliers are positive. Derivatives with respect to resources are as follows:

1) Derivative w.r.t. $r_{j}$ for users $j \in M S_{R S i} \cap U_{D}$, $\phi=B S, R S$ :

$$
\begin{aligned}
\frac{\partial L\left(\mathbf{w}, \mathbf{p}, \mathbf{T}_{i}, \overline{\lambda_{p}}, \overline{\lambda_{w}}, \overline{\lambda_{r}}, \lambda_{T}\right)}{\partial r_{j}}=0 \\
\Rightarrow\left[\frac{1}{\lambda_{j}^{r, B S}+\lambda_{j}^{r, R S}}-\frac{\alpha R_{j}}{1-\alpha}\right]^{+}=r_{j}
\end{aligned}
$$


2) Derivative w.r.t. $w_{j}^{\phi}$ and $p_{j}^{\phi}$ for users $j \in M S_{R S i}, \phi=$ $B S, R S$ :

$$
\begin{gathered}
\frac{\partial L\left(\mathbf{w}, \mathbf{p}, \mathbf{T}_{i}, \overline{\lambda_{p}}, \overline{\lambda_{w}}, \overline{\lambda_{r}}, \lambda_{T}\right)}{\partial w_{j}^{\phi}}=0 \\
\Rightarrow T_{i}^{\phi}\left(\log \left(1+\frac{p_{j}^{\phi}}{n_{j}^{\phi} w_{j}^{\phi}}\right)-\frac{\frac{p_{j}^{\phi}}{n_{j}^{\phi} w_{j}^{\phi}}}{1+\frac{p_{j}^{\phi}}{n_{j}^{\phi} w_{j}^{\phi}}}\right)=\frac{\lambda_{w}^{\phi}}{\lambda_{j}^{r, \phi}} \\
\frac{\partial L\left(\mathbf{w}, \mathbf{p}, \mathbf{T}_{i}, \overline{\lambda_{p}}, \overline{\lambda_{w}}, \overline{\lambda_{r}}, \lambda_{T}\right)}{\partial p_{j}^{\phi}}=0 \Rightarrow \frac{T_{i}^{\phi}}{n_{j}^{\phi}\left(1+\frac{p_{j}^{\phi}}{n_{j}^{\phi} w_{j}^{\phi}}\right)}=\frac{\lambda_{p}^{\phi}}{\lambda_{j}^{r, \phi}}
\end{gathered}
$$

Dividing Eq. (22) to (23) we get the following relation:

$$
\frac{\lambda_{x}^{\phi}}{n_{j}^{\phi}}=\frac{\lambda_{w}^{\phi}}{n_{j}^{\phi} \lambda_{p}^{\phi}}=\left(1+\frac{p_{j}^{\phi}}{n_{j}^{\phi} w_{j}^{\phi}}\right) \log \left(1+\frac{p_{j}^{\phi}}{n_{j}^{\phi} w_{j}^{\phi}}\right)-\frac{p_{j}^{\phi}}{n_{j}^{\phi} w_{j}^{\phi}}
$$

Let's define $\lambda_{x}^{\phi}=\lambda_{w}^{\phi} / \lambda_{p}^{\phi}$ and the function $f_{x}(x)=(1+$ $x) \log (1+x)-x$. This is a monotonic increasing and convex function. Using $\lambda_{x}^{\phi}(i)$ we can find the scaled SINR $x_{j}^{\phi}=\frac{p_{j}^{\phi}}{n_{i}^{\phi} w_{j}^{\phi}}$ as $f_{x}^{-1}\left(\lambda_{x}^{\phi} / n_{j}^{\phi}\right)$.

3) Derivative w.r.t. $T_{i}^{\phi}$, for $\phi=B S, R S$ :

$$
\begin{aligned}
& \frac{\partial L\left(\mathbf{w}, \mathbf{p}, \mathbf{T}_{i}, \overline{\lambda_{p}}, \overline{\lambda_{w}}, \overline{\lambda_{r}}, \lambda_{T}\right)}{\partial T_{i}^{\phi}}=0 \\
& \Rightarrow \sum_{j \in M S_{R S i}} \lambda_{j}^{r, \phi} w_{j}^{\phi} \log \left(1+\frac{p_{j}^{\phi}}{n_{j}^{\phi} w_{j}^{\phi}}\right)=\lambda_{T}
\end{aligned}
$$

Using Equations (25), (23) and (24) we can write,

$$
\begin{aligned}
\lambda_{T} & =\sum_{j \in M S_{R S i}} \frac{\lambda_{p}^{\phi}}{T_{i}^{\phi}} n_{j}^{\phi} w_{j}^{\phi}\left(1+\frac{p_{j}^{\phi}}{n_{j}^{\phi} w_{j}^{\phi}}\right) \log \left(1+\frac{p_{j}^{\phi}}{n_{j}^{\phi} w_{j}^{\phi}}\right) \\
\lambda_{T} & =\sum_{j \in M S_{R S i}} \frac{\lambda_{p}^{\phi}}{T_{i}^{\phi}} n_{j}^{\phi} w_{j}^{\phi}\left(\frac{\lambda_{w}^{\phi}}{\lambda_{p}^{\phi} n_{j}^{\phi}}+\frac{p_{j}^{\phi}}{n_{j}^{\phi} w_{j}^{\phi}}\right) \\
\lambda_{T} & =\sum_{j \in M S_{R S i}}\left(\frac{w_{j}^{\phi} \lambda_{w}^{\phi}}{T_{i}^{\phi}}+\frac{\lambda_{p}^{\phi} p_{j}^{\phi}}{T_{i}^{\phi}}\right) \\
T_{i}^{\phi} & =\frac{W \lambda_{w}^{\phi}+P^{\phi} \lambda_{p}^{\phi}}{\lambda_{T}}
\end{aligned}
$$

Using Equation (23) and (26) we get:

$$
\begin{aligned}
\lambda_{j}^{r, \phi} & =\lambda_{T} \lambda_{p}^{\phi} \frac{n_{j}^{\phi}\left(1+f_{x}^{-1}\left(\frac{\lambda_{x}^{\phi}}{n_{j}^{\phi}}\right)\right)}{W \lambda_{w}^{\phi}+P^{\phi} \lambda_{p}^{\phi}} \\
& =\lambda_{T} \frac{n_{j}^{\phi}\left(1+f_{x}^{-1}\left(\frac{\lambda_{x}^{\phi}}{n_{j}^{\phi}}\right)\right.}{W \lambda_{x}^{\phi}+P^{\phi}}
\end{aligned}
$$

Combining (21) and (27) we obtain the rate function for data users in terms of $\lambda_{x}^{B S}, \lambda_{x}^{R S}$ and $\lambda_{T}$ as,

$$
\begin{aligned}
& r_{j}\left(\lambda_{x}^{B S}, \lambda_{x}^{R S}, \lambda_{T}\right)= \\
& \left(\frac{1}{\frac{\lambda_{T} n_{j}^{B S}\left(1+f_{x}^{-1}\left(\frac{\lambda_{x}^{B S}}{n_{j}^{B S}}\right)\right)}{W \lambda_{x}^{B S}+P^{B S}}+\frac{\lambda_{T} n_{j}^{R S}\left(1+f_{x}^{-1}\left(\frac{\lambda_{x}^{R S}}{n_{j}^{R S}}\right)\right)}{W \lambda_{x}^{R S}+P^{R S}}}-\widetilde{\alpha} R_{j}\right)
\end{aligned}
$$

Taking the derivative of (20) w.r.t. $\lambda_{p}^{\phi}$ we obtain the power constraints and combining it with (24) we obtain,

$$
P^{\phi}=\sum_{j \in M S_{R S i}} f_{x}^{-1}\left(\frac{\lambda_{x}^{\phi}}{n_{j}^{\phi}}\right) n_{j}^{\phi} w_{j}^{\phi}, \phi=B S, R S
$$

Since $n_{j}^{B S}=n^{B S}$ for $j \in M S_{R S i}$ (they are all $B S-R S_{i}$ channel condition), SINR's in those links are the same using (24). Therefore optimal SINRs of all users in the BS-RS links are equal to $x^{B S *}=f_{x}^{-1}\left(\frac{\lambda_{x}^{B S *}}{n^{B S}}\right)=\frac{P^{B S}}{n^{B S W}}$. After some arrangements in (28) we can write the rates of all data sessions as a function of only $\lambda_{x}^{R S}$ and $\lambda_{T}$ as follows:

$$
r_{j}\left(\lambda_{x}^{R S}, \lambda_{T}\right)=\left(\frac{W / \lambda_{T}}{\frac{1}{\log \left(1+\frac{P B S}{n^{B S}}\right)}+\frac{\left(1+f_{x}^{-1}\left(\frac{\lambda_{x}^{R S}}{n_{j}^{R S}}\right)\right)}{\frac{\lambda_{x}^{R S}}{n_{j}^{R S}}+\frac{P R S}{n_{j}^{R S}}}-\widetilde{\alpha} R_{j}}\right)^{+}
$$

Rate $r_{j}\left(\lambda_{x}^{R S}, \lambda_{T}\right)$ is a nonincreasing function of $\lambda_{x}^{R S}$ for $0 \leq \lambda_{x}^{R S} \leq n_{j}^{R S} f_{x}\left(\frac{P^{R S}}{n_{j}^{R S} W}\right)$ and it is a nondecreasing function of $\lambda_{x}^{R S}$ for $n_{j}^{R S} f_{x}\left(\frac{P^{R S}}{n_{j}^{R S} W}\right) \leq \lambda_{x}^{R S}$. For finite $\lambda_{T}$ it always takes finite values.

4) Calculation of times: Sum of the time-bandwidth products in BS-RS and RS-MS composite links is as follows,

$$
\begin{aligned}
& \sum_{j \in M S_{R S i}} T_{i}^{\phi}\left(\lambda_{x}^{R S}, \lambda_{T}\right) w_{j}^{\phi}=\sum_{j \in M S_{R S i} \cap U_{D}} \frac{r_{j}\left(\lambda_{x}^{R S}, \lambda_{T}\right)}{\log \left(1+f_{x}^{-1}\left(\frac{\lambda_{x}^{\phi}}{n_{j}^{\phi}}\right)\right)} \\
& +\sum_{j \in M S_{R S i} \cap U_{R}} \frac{r_{j}^{c, \phi}}{\log \left(1+f_{x}^{-1}\left(\frac{\lambda_{x}^{\phi}}{n_{j}^{\phi}}\right)\right)}
\end{aligned}
$$

Each node in a composite link transmits using the same time interval, but different frequency bands, where the sum of the bandwidths is equal to $W$. Dividing the total time-bandwidth product to $W$ we can find the time intervals allocated to BS-RS 
and RS-MS composite links:

$$
\begin{aligned}
T_{i}^{B S}\left(\lambda_{x}^{R S}, \lambda_{T}\right)= & \sum_{j \in M S_{R S i} \cap U_{D}} \frac{r_{j}\left(\lambda_{x}^{R S}, \lambda_{T}\right)}{W \log \left(1+\frac{P^{B S}}{n^{B S} W}\right)} \\
+\sum_{j \in M S_{R S i} \cap U_{R}} \frac{r_{j}^{c, B S}}{W \log \left(1+\frac{P^{B S}}{n^{B S} W}\right)} & \\
T_{i}^{R S}\left(\lambda_{x}^{R S}, \lambda_{T}\right)= & \sum_{j \in M S_{R S i} \cap U_{D}} \frac{r_{j}\left(\lambda_{x}^{R S}, \lambda_{T}\right)}{W \log \left(1+f_{x}^{-1}\left(\frac{\lambda_{x}^{R S}}{n_{j}^{R S}}\right)\right)} \\
& \left.+\sum_{j \in M S_{R S i} \cap U_{R}}^{c, R S} \frac{r_{j}^{\prime}}{W \log \left(1+f_{x}^{-1}\left(\frac{\lambda_{x}^{R S}}{n_{j}^{R S}}\right)\right.}\right)
\end{aligned}
$$

5) Calculation of total power: Sum of the powers in BSRS and RS-MS transmissions can be found as follows:

$$
\begin{aligned}
S_{p}^{B S}\left(\lambda_{x}^{R S}, \lambda_{T}\right) & =\sum_{j \in M S_{R S i} \cap U_{D}} \frac{r_{j}\left(\lambda_{x}^{R S}, \lambda_{T}\right) n^{B S} \frac{P^{B S}}{n^{B S} W}}{T_{i}^{B S}\left(\lambda_{x}^{R S}, \lambda_{T}\right) \log \left(1+\frac{P^{B S}}{n^{B S} W}\right)} \\
+ & \sum_{j \in M S_{R S i} \cap U_{R}} \frac{r_{j}^{c, B S} n^{B S} \frac{P^{B S}}{n^{B S} W}}{T_{i}^{B S}\left(\lambda_{x}^{R S}, \lambda_{T}\right) \log \left(1+\frac{P^{B S}}{n^{B S} W}\right)} \\
S_{p}^{R S}\left(\lambda_{x}^{R S}, \lambda_{T}\right) & \left.=\sum_{j \in M S_{R S i} \cap U_{D}} \frac{r_{j}\left(\lambda_{x}^{R S}, \lambda_{T}\right) n_{j}^{R S} f_{x}^{-1}\left(\frac{\lambda_{x}^{R S}}{n_{j}^{R S}}\right)}{\left.\lambda_{x}^{R S}, \lambda_{T}\right) \log \left(1+f_{x}^{-1}\left(\frac{\lambda_{x}^{R S}}{n_{j}^{R S}}\right)\right.}\right) \\
+\sum_{j \in M S_{R S i} \cap U_{R}} & \frac{r_{j}^{c, R S} n_{j}^{R S} f_{x}^{-1}\left(\frac{\lambda_{x}^{R S}}{n_{j}^{R S}}\right)}{T_{i}^{R S}\left(\lambda_{x}^{R S}, \lambda_{T}\right) \log \left(1+f_{x}^{-1}\left(\frac{\lambda_{x}^{R S}}{n_{j}^{R S}}\right)\right)}
\end{aligned}
$$

Our objective is to find the resource allocation such that

$$
\begin{aligned}
T_{i}^{B S}\left(\lambda_{x}^{R S}, \lambda_{T}\right)+ & T_{i}^{R S}\left(\lambda_{x}^{R S}, \lambda_{T}\right)=T_{i} \\
S_{p}^{R S}\left(\lambda_{x}^{R S}, \lambda_{T}\right) & =P^{R S} \\
S_{p}^{B S}\left(\lambda_{x}^{R S}, \lambda_{T}\right) & =P^{B S}
\end{aligned}
$$

In fact the equations (32) and (34) are equivalent. Arranging (35) and putting $T_{i}^{R S}\left(\lambda_{T}, \lambda_{x}^{R S}\right)$ in the left hand side; and considering (33) we get the following equation:

$$
\begin{aligned}
& \sum_{j \in M S_{R S i} \cap U_{D}} \frac{r_{j}\left(\lambda_{x}^{R S}, \lambda_{T}\right)}{W \log \left(1+f_{x}^{-1}\left(\frac{\lambda_{x}^{R S}}{n_{j}^{R S}}\right)\right)} \\
& +\sum_{j \in M S_{R S i} \cap U_{R}} \frac{r_{j}^{c, R S}}{W \log \left(1+f_{x}^{-1}\left(\frac{\lambda_{x}^{R S}}{n_{j}^{R S}}\right)\right)} \\
& =\sum_{j \in M S_{R S i} \cap U_{D}} \frac{r_{j}\left(\lambda_{x}^{R S}, \lambda_{T}\right) n_{j}^{R S} f_{x}^{-1}\left(\frac{\lambda_{x}^{R S}}{n_{j}^{R S}}\right)}{P^{R S} \log \left(1+f_{x}^{-1}\left(\frac{\lambda_{x}^{R S}}{n_{j}^{R S}}\right)\right)} \\
& +\sum_{j \in M S_{R S i} \cap U_{R}} \frac{r_{j}^{c, R S} n_{j}^{R S} f_{x}^{-1}\left(\frac{\lambda_{x}^{R S}}{n_{j}^{R S}}\right)}{P^{R S} \log \left(1+f_{x}^{-1}\left(\frac{\lambda_{x}^{R S}}{n_{j}^{R S}}\right)\right)}
\end{aligned}
$$

Then the following equality should hold at the optimal $\lambda_{x}^{R S *}$ and $\lambda_{T}$

$$
\begin{aligned}
\sum_{j \in M S_{R S i} \cap U_{D}} & r_{j}\left(\lambda_{x}^{R S}, \lambda_{T}\right)\left(A_{j}\left(\lambda_{x}^{R S}\right)-B_{j}\left(\lambda_{x}^{R S}\right)\right) \\
& +\sum_{j \in M S_{R S i} \cap U_{R}} r_{j}^{c, R S}\left(A_{j}\left(\lambda_{x}^{R S}\right)-B_{j}\left(\lambda_{x}^{R S}\right)\right)=0
\end{aligned}
$$

where

$$
\begin{aligned}
A_{j}\left(\lambda_{x}^{R S}\right) & =\frac{1}{W \log \left(1+f_{x}^{-1}\left(\frac{\lambda_{x}^{R S}}{n_{j}^{R S}}\right)\right)} \\
B_{j}\left(\lambda_{x}^{R S}\right) & =\frac{1}{W \log \left(1+f_{x}^{-1}\left(\frac{\lambda_{x}^{R S}}{n_{j}^{R S}}\right)\right)} \frac{f_{x}^{-1}\left(\frac{\lambda_{x}^{R S}}{n_{j}^{R S}}\right)}{\frac{P^{R S}}{n_{j}^{R S} W}}
\end{aligned}
$$

$A_{j}\left(\lambda_{x}^{R S}\right)$ is a decreasing and $B_{j}\left(\lambda_{x}^{R S}\right)$ is an increasing function of $\lambda_{x}^{R S}$.

Lemma 1: Left hand side of (40) is a monotonic nonincreasing function of $\lambda_{x}^{R S}$ that decreases from $+\infty$ to $-\infty$ and crosses zero at a single point.

Proof: We will start the analysis from a single user. For a data user $j$ and for $\lambda_{T}>0$, the function $r_{j}\left(\lambda_{x}^{R S}, \lambda_{T}\right)$ takes finite values for all $0<\lambda_{x}^{R S}$. It is a either zero or a decreasing function of $\lambda_{x}^{R S}$ for $\lambda_{x}^{R S}<n_{j}^{R S} f_{x}\left(\frac{P^{R S}}{n_{j}^{R S} W}\right)$ and either zero or increasing function of $\lambda_{x}^{R S}$ for $\lambda_{x}^{R S}>n_{j}^{R S} f_{x}\left(\frac{P^{R S}}{n_{j}^{R S} W}\right)$. For real time users rate function is constant.

It can also easily be shown that $A_{j}\left(\lambda_{x}^{R S}\right)-B_{j}\left(\lambda_{x}^{R S}\right)$ is a decreasing function of $\lambda_{x}^{R S}$ which takes positive values for $\lambda_{x}^{R S}<n_{j}^{R S} f_{x}\left(\frac{P^{R S}}{n_{j}^{R S} W}\right)$ and negative values for $\lambda_{x}^{R S}>n_{j}^{R S} f_{x}\left(\frac{P^{R S}}{n_{j}^{R S} W}\right)$. For $\lambda_{x}^{R S}<n_{j}^{R S} f_{x}\left(\frac{P^{R S}}{n_{j}^{R S} W}\right)$ the LHS of (40) is a product of two positive decreasing functions and it is decreasing for user $\mathrm{j}$. For $\lambda_{x}^{R S}>n_{j}^{R S} f_{x}\left(\frac{P^{R S}}{n_{j}^{R S} W}\right)$ it is the product of a positive increasing and negative decreasing function hence it is also decreasing in this region for user $\mathrm{j}$. Hence, LHS of (40), summation of such functions for all users is a monotonic decreasing function 
Let $\lambda_{x}^{R S *}\left(\lambda_{T}\right)$ be the Lagrangian multiplier that satisfies Equation (40) (Please note that the power constraint is automatically satisfied for BS-RS by setting $x_{j}^{B S}=\frac{P^{B S}}{W n^{B S}}$ for $j \in M S_{R S i}$ ). Since the LHS of Equation (40) is a monotonic function this value can be found by a simple binary search. Then $T_{i}^{B S}\left(\lambda_{x}^{R S *}\left(\lambda_{T}\right), \lambda_{T}\right)$ and $T_{i}^{R S}\left(\lambda_{x}^{R S *}\left(\lambda_{T}\right), \lambda_{T}\right)$ become the corresponding time allocated to BS-RS and RSMS transmissions. We are looking for the Lagrange multiplier values $\left(\lambda_{x}^{R S *}\left(\lambda_{T}^{*}\right), \lambda_{T}^{*}\right)$ that satisfies both (40) and $T_{i}^{B S}\left(\lambda_{x}^{R S *}\left(\lambda_{T}^{*}\right), \lambda_{T}^{*}\right)+T_{i}^{R S}\left(\lambda_{x}^{R S *}\left(\lambda_{T}^{*}\right), \lambda_{T}^{*}\right)=T_{i}$. Since rate is a monotonic function of $\lambda_{T}$ total time is also monotonic from (32) and (33). We need another binary search on $\lambda_{T}$. Hence we can find the optimal power,bandwidth and time by two nested binary searches. The algorithm will be described in more detail.

\section{Algorithm}

Main Algorithm:

1) Determine required rates for real time sessions

2) Test feasibility: If $\underline{T}_{i}^{B S}+\underline{T}_{i}^{R S}>T_{i}$ (using (13)) then find the link with non-zero real time session rate and worst channel condition and drop it. Repeat this step until feasibility is reached.

3) $\operatorname{Run}\left(\mathbf{T}, \lambda_{b}^{*}\right)=$ BinarySearchCelllular ()

4) For all microcells

Run $\left(\lambda_{x}^{R S *}, \lambda_{T}^{*}\right)=$ BinarySearchMicrocell ()

Procedure: $\left(\left(\mathbf{T}, \lambda_{b}^{*}\right)\right)=$ BinarySearchCellular () : Finds the $\lambda_{b}^{*}$ so that $S_{b}\left(\lambda_{b}^{*}\right)=W T_{f}$.

1) Find the smallest $k$ such that $S_{b}\left(2^{k} \Delta \lambda_{b}\right)<W T_{f}$ in (11). Set $\lambda_{b}^{h}=2^{k} \Delta \lambda_{b}, \lambda_{b}^{l}=2^{k-1} \Delta \lambda_{b}$.

Repeat Step 2 until $\left|\frac{S_{b}\left(\lambda_{b}^{m}\right)}{W T_{f}}-1\right|<\varepsilon$

2) Set $\lambda_{b}^{m}=\left(\lambda_{b}^{l}+\lambda_{b}^{h}\right) / 2$. If $S_{b}\left(\lambda_{b}^{m}\right)<W T_{f}$ then $\lambda_{b}^{h}=\lambda_{b}^{m}$ else $\lambda_{b}^{l}=\lambda_{b}^{m}$

3) $\lambda_{b}^{*}=\lambda_{b}^{m}$. Calculate microcell times $T_{i}=T_{i}^{R S}\left(\lambda_{b}^{*}\right)+$ $T_{i}^{B S}\left(\lambda_{b}^{*}\right)$ according to (12) for all $i \in M C$.

Procedure $:\left(\lambda_{x}^{R S *}, \lambda_{T}^{*}\right)=$ BinarySearchTime () :

1) Run BinarySearch $x_{x}^{R S}\left(2^{k} \Delta \lambda_{T}\right)$ and find the smallest $k$ such that $T_{i}^{B S}\left(\lambda_{x}^{R S *}\left(2^{k} \Delta \lambda_{T}\right), 2^{k} \Delta \lambda_{T}\right)+$ $T_{i}^{R S}\left(\lambda_{x}^{R S *}\left(2^{k} \Delta \lambda_{T}\right), 2^{k} \Delta \lambda_{T}\right)>T_{i}$. Set $\lambda_{T}^{h}=2^{k} \Delta \lambda_{T}$, $\lambda_{T}^{l}=2^{k-1} \Delta \lambda_{T}$.

Repeat Step 2 until $\left|\frac{T_{i}}{T_{i}^{B S *}+T_{i}^{B S *}}-1\right|<\varepsilon$

2) Set $\lambda_{T}^{m}=\left(\lambda_{T}^{h}+\lambda_{T}^{l}\right) / 2$ and run $\lambda_{x}^{R S *}\left(\lambda_{T}^{m}\right)=$ BinarySearch ${ }_{x}^{R S}\left(\lambda_{T}^{m}\right)$.

- If $T_{i}^{B S}\left(\lambda_{x}^{R S *}\left(\lambda_{T}^{m}\right), \lambda_{T}^{m}\right)+T_{i}^{R S}\left(\lambda_{x}^{R S *}\left(\lambda_{T}^{m}\right), \lambda_{T}^{m}\right)>T_{i}$ then $\lambda_{T}^{l}=\lambda_{T}^{m}$.

- else $\lambda_{T}^{h}=\lambda_{T}^{m}$.

3) Set $\lambda_{T}^{*}=\lambda_{T}^{m}$ and $\lambda_{x}^{R S *}=\lambda_{x}^{R S *}\left(\lambda_{T}^{m}\right)$. Using these values compute the optimal time allocations $T_{i}^{R S *}, T_{i}^{B S *}, \forall i \in M C$ and $p_{j}^{\phi}, w_{j} \phi, \forall j \in U, \phi=B S, R S$

Procedure $: \lambda_{x}^{R S *}\left(\lambda_{T}\right)=$ BinarySearch ${ }_{x}^{R S}\left(\lambda_{T}\right):$ Finds the

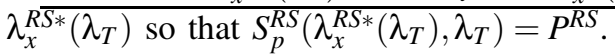

1) Find the smallest $k$ such that $S_{p}^{R S}\left(2^{k} \Delta \lambda_{x}^{R S}, \lambda_{T}\right)>P^{R S}$. Set $\lambda_{x}^{R S, h}=2^{k} \Delta \lambda_{x}^{R S}, \lambda_{x}^{R S, l}=2^{k-1} \Delta \lambda_{x}^{R S}$.
Repeat Step 2 until $\left|\frac{S_{p}\left(\lambda_{x}^{R S, m}, \lambda_{T}\right)}{P^{R S}}-1\right|<\varepsilon$

2) Set $\lambda_{T}^{m}=\left(\lambda_{T}^{l}+\lambda_{T}^{h}\right) / 2$. If $S_{p}^{R S}\left(\lambda_{x}^{R S, m}, \lambda_{T}\right)<P^{R S}$ then $\lambda_{T}^{l}=$ $\lambda_{T}^{m}$ else $\lambda_{T}^{h}=\lambda_{T}^{m}$

Figure 3 shows a typical binary search process for a microcell. At each step a $\left(\lambda_{T}, \lambda_{x}^{R S}\left(\lambda_{T}\right)\right)$ pair is found such that the sum of powers is equal to $P^{R S}$. Since for such pairs time $T_{i}^{R S}+T_{i}^{B S}$ is monotonic decreasing in $\lambda_{T}$ (as seen in the figure), we are able to find the optimal $\lambda_{T}$ by a binary search. Since the channel condition in the access (BS-RS) link is usually much better, usually $T_{i}^{B S}<T_{i}^{R S}$. In this example time slot length is $0.1 \mathrm{msec}$, and after the optimization, all times will be rounded to this value. Therefore we can stop the search when we come less than $0.05 \mathrm{msec}$ close to the time constraint (which is $2 \mathrm{msec}$ in this example)

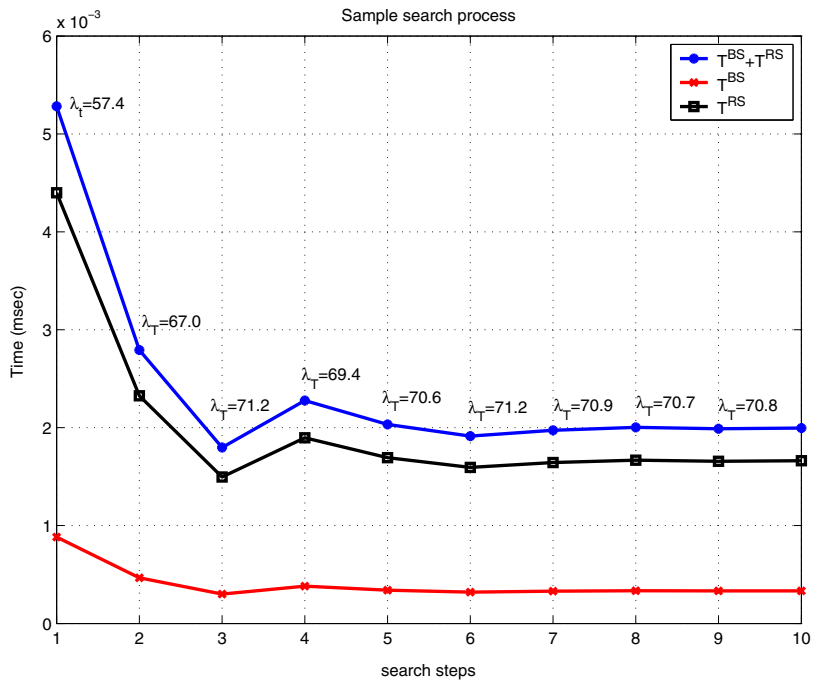

Fig. 3. A sample binary search process

\section{NUMERICAL EVALUATION}

Figure 4 shows a sample MR system. We consider a tandem network of $2 \mathrm{~km}$ radius, where the BS is at the $(0,0)$ coordinate. The RSs are located at $1400 \mathrm{~m}$ to the end of the MR-cell. MSs are located at 400,800,1200,1600 and 2000 meters. In order to make the station assignment, all the stations (BS and RSs) send broadcast signals (Transmission power for the BS and RSs is $P^{B S}$ and $P^{R S}$, respectively). Each MS is assigned to the station (either the BS or one of the RSs) that maximizes the received power. ${ }^{2}$

As for the path loss, we use the IEEE 802.16j channel model proposed in [11]. For $B S \rightarrow M S$ and $R S \rightarrow M S$ we use the Nonline-of-sight (NLOS) and for $B S \rightarrow R S$ and we use the LOS model. We assume log-normal fading with variance equal to $8 \mathrm{Db}$ for $\mathrm{BS}$ transmissions and $3.1 \mathrm{~dB}$ for RS transmissions, and Rayleigh fading with mean equal to 0.6. We assume that

\footnotetext{
${ }^{2}$ In a real system RSs can be located according to the user density in the MR cell area. Such a topology design problem is beyond the scope of this paper.
} 


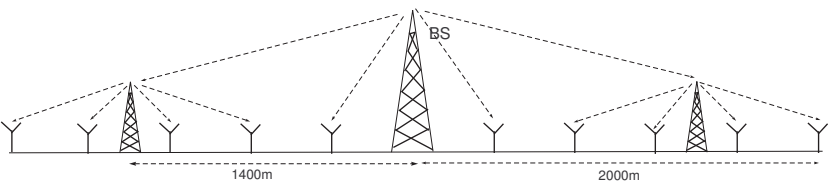

Fig. 4. A sample MR model for numerical evaluation

\begin{tabular}{|c|c|}
\hline Parameter & Value \\
\hline \hline Cell radius & $2 \mathrm{~km}$ \\
\hline User Distances & $0.4,0.8,1.2,1.6,2.0 \mathrm{~km}$ \\
\hline RS Distance & $1.4 \mathrm{~km}$ \\
\hline \# microcells $(\mathrm{M})$ & 3 \\
\hline BS,RS Power $\left(P^{B S}, P^{R S}\right)$ & $(20,5) \mathrm{W}$ \\
\hline$W_{s} u b, N_{\text {sub }}$ & $267 \mathrm{KHz}, 30$ \\
\hline Frame Length $T_{f}$ & $2 \mathrm{msec}$ \\
\hline Slot Length $T_{s}$ & $0.1 \mathrm{msec}$ \\
\hline Voice Traffic & CBR $32 \mathrm{kbps}$ \\
\hline Video Traffic & $802.16-128 \mathrm{kbps}$ \\
\hline FTP File & $5 \mathrm{MB}$ \\
\hline AWGN p.s.d.( $\left(N_{0}\right)$ & $-174 \mathrm{dBm} / \mathrm{Hz}$ \\
\hline Coherent Time (Fast/Slow) & $(4 \mathrm{msec} / 400 \mathrm{msec})$. \\
\hline BS-RS PL(d)(in dB) & $36.5+23.5 \log _{10} d+\psi_{d B}^{B S-R S}$ \\
\hline RS-MS PL(d)(in dB) & $31.5+35 \log _{10} d+\psi_{d B}^{R S-M S}$ \\
\hline BS-MS PL(d)(in dB) & $31.5+35 \log _{10} d+\psi_{d B}^{B S-M S}$ \\
\hline$\psi_{d B}^{B S-M S}, \psi_{d B}^{R S-M S}$ & $\sim N(0 d B, 8 d B)$ \\
\hline$\psi_{d B}^{B S-R S}$ & $\sim N(0 d B, 3.1 d B)$ \\
\hline
\end{tabular}

TABLE I

SimUlation PARAMETERS

rayleigh fading stays constant at each frame and log-normal fading stays constant during 5 frames. Frame length is equal to 20 slots and each slot is $T_{s}=1 \mathrm{msec}$. Base station and each relay has $P^{B S}=20 \mathrm{~W}$ and $P^{R S}=5 \mathrm{Ws}$ of power, respectively. Bandwidth is equal to $B=10 \mathrm{MHz}$.

Our traffic model is based on [12], and it is as follows: For each data (FTP) session we assume a single 5MB file arriving at the queue at time zero. We assume $32 \mathrm{kbps}$ VoIP sessions, where a 320-bit packet arrives at every 10 time slots. Finally we assume $128 \mathrm{kbps}$ video streaming sessions, with a fixed video frame duration of $100 \mathrm{msec}$. During each frame there are 8 packets (slices). Packet size is Truncated Pareto distributed with certain min, max and shape parameters. Interarrival time between packets is also Truncated Pareto distributed with certain min, max and shape parameters such that all packets arrive during a $100 \mathrm{~ms}$ frame. We assume that bits arrive at the end of a time slot and they are ready to transmit at the beginning of the next time slot. Performance Criteria are

1) 95 percentile delay for voice sessions

2) 95 percentile delay for video sessions

3) Total and log-sum throughput for data sessions

Keeping the number of data and voice users at 20 each, we vary the number of video user from 20 to 50 . Figure 5 shows the $95^{\text {th }}$ percentile delay for voice sessions. We can observe that in the 2-RS system users at all distances delay stays under the required $100 \mathrm{msec}$ level, while for the system with no RSs, users at $1.6 \mathrm{~km}$ and $2.0 \mathrm{~km}$ experience severe delays. Since the coherence time for the log-normal fading is much longer than the voice delay constraint, delays for edge users by far exceed the required levels.

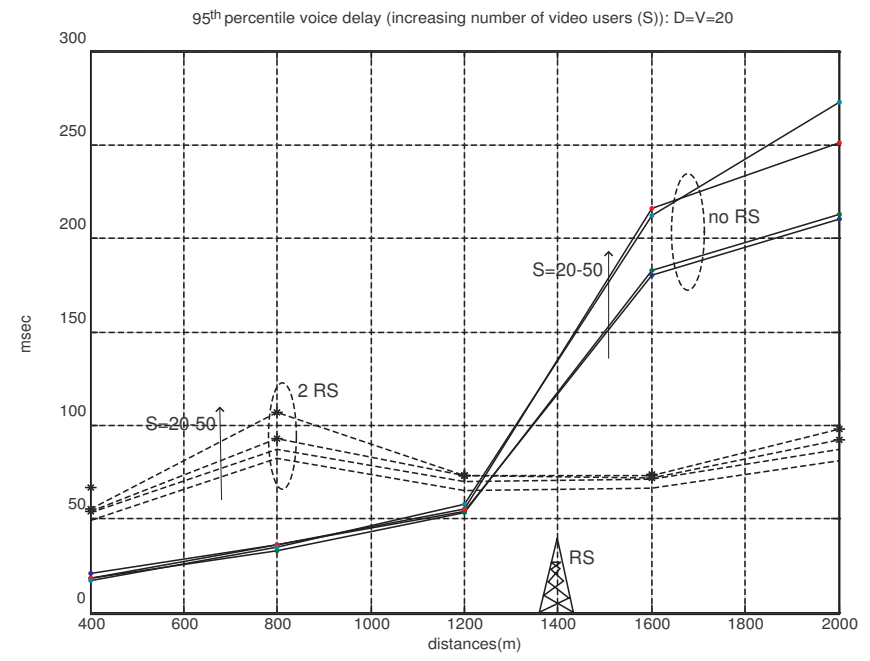

Fig. 5. $95^{\text {th }}$ percentile voice delay vs. distance to the BS for increasing number of video sessions.

Figure 6 shows the $95^{\text {th }}$ percentile delay for video sessions. We again observe that using relays we can prevent QoS violation for users at all distance levels in the cell. Without RSs, users at the cell edge experience high delays.

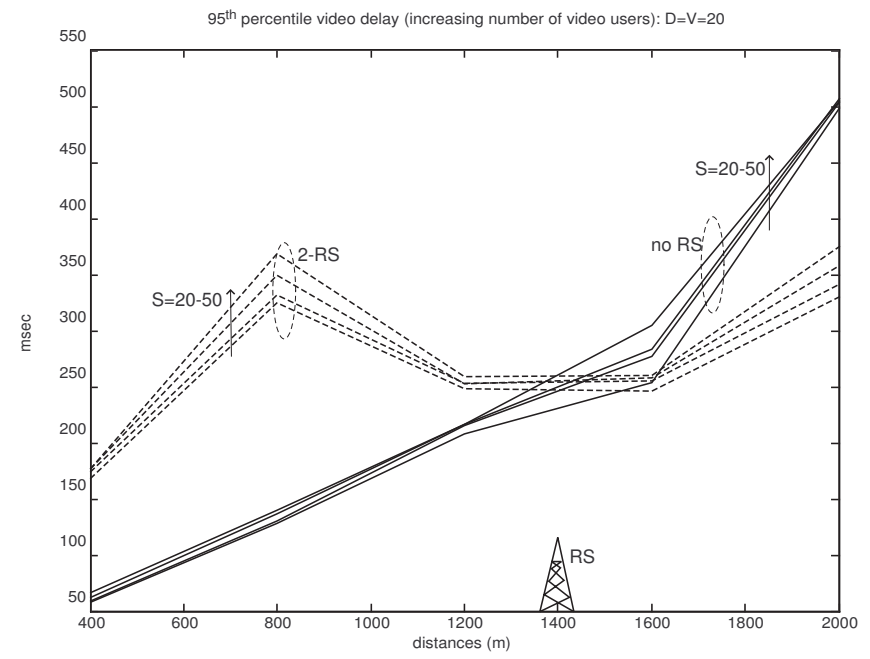

Fig. 6. $95^{\text {th }}$ percentile video delay vs. distance to the BS for increasing number of video sessions.

Figure 7 shows the total throughput for users at different distance levels. Here we observe the negative effect of using relays on throughput. Sessions in the RS-microcells have to 
travel two links. These two links are both very likely to experience a better channel condition than a single BS-MS link, however transmission of a packet requires two frames. Because of this trade-off be observe from Figure 7 that users at $0.4,0.8 \mathrm{~km}$ receive more throughput in the $0-\mathrm{RS}$ case. On the other hand users at 1.2, 1.6 and $2.0 \mathrm{~km}$ receive more throughput in the 2-RS system.

We also observe that total throughput decreases more with increasing number of video users in the 0-RS case. In the 2RS case a video user takes less throughput. Therefore in the case of large number of video users, a system with relays is expected to provide more throughput to data users.

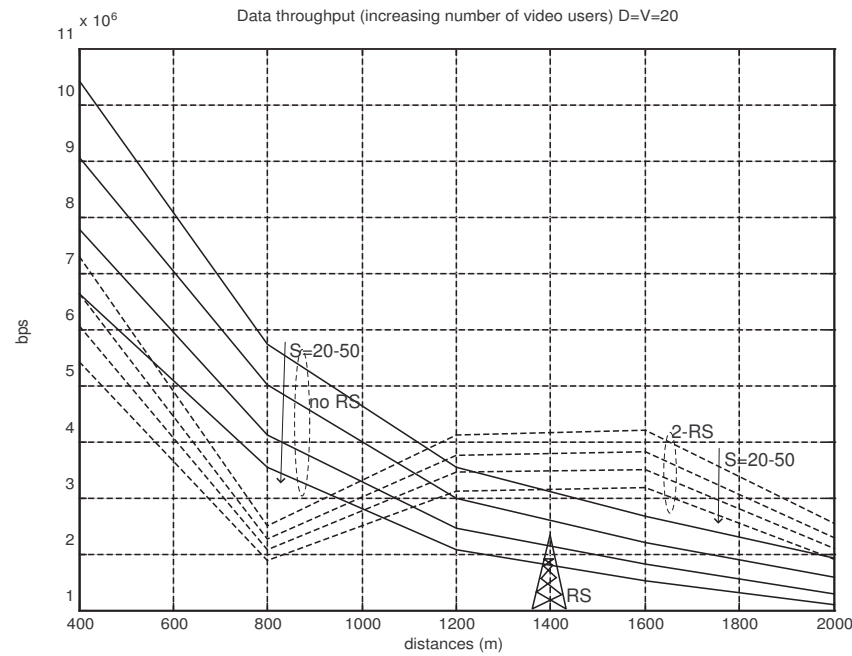

Fig. 7. Total throughput of data users vs. distance to the BS for increasing number of video sessions.
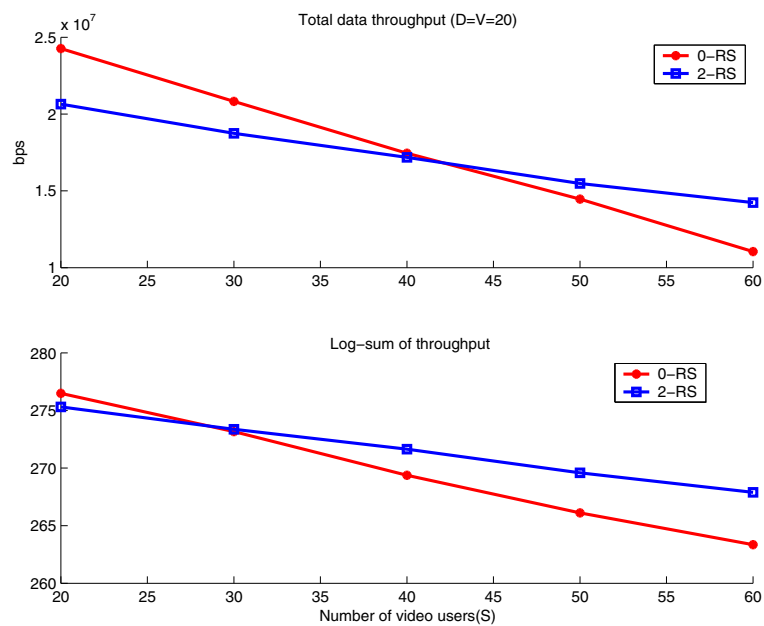

Fig. 8. Total throughput and log-sum of throughput of data users vs. number of video sessions.

We observe in Figure 8 the trade-off between SINR improvement by RSs and time loss by relaying. For low number of video users 0-RS system provides better throughput and proportional fairness. However a video session costs more resources in a 0-RS system; total throughput decreases more with increasing number of video users in the 0-RS case. Therefore in the case of large number of video users $(S>40)$, a system with relays is expected to provide more throughput to data users. Also for number of video users greater than 30 , relay system provides better proportional fairness.

\section{CONCLUSIONS}

In this work we proposed a joint time, power and bandwidth allocation scheme for downlink transmission in the presence of single-interface relay stations. The proposed scheme consists of two steps, namely subframe allocation for each microcell and joint time, power,bandwidth allocation for links in each microcell. Numerical results show that it is possible to increase the cell size and decrease the number of base stations by adding low-cost relay stations. Multihop relay systems satisfy the QoS requirements of all real time sessions, for the cases, in which regular cellular systems are not sufficient. Future work will include relay station positioning, frequency reuse for relays and usage of cooperative diversity techniques multihop relay networks.

\section{REFERENCES}

[1] IEEE 802.16 2004. Amendment to IEEE Standard for Local and Metropolitan Area Networks - Part 16: Air Interface for Fixed Broadband Wireless Access Systems. IEEE, Oct. 2004.

[2] IEEE 802.16e. IEEE Standard for Local and Metropolitan Area Networks, Part 16: Air Interface for Fixed and Mobile Broadband Wireless Access Systesm, Amendment 2: Physical and Medium Access Control Layers for Combined Fixed and Mobiel Operation in Licensed Bands and Corrigendum 1. IEEE, Feb. 2006.

[3] A. Ghosh, D. Wolter, J. G. Andres, and R Chen. Broadband Wireless Access with WiMax/802.16: Current Performance Benchmarks and Future Potential. IEEE Communications Magazine, Feb. 2005.

[4] C. Eklund, R. B. Marks, K.L. Stanwood, and S. Wang. IEEE Standard 802.16: A Technical Overview of the WirelessMAN Air Interface for Broadband Wireless Access. IEEE Communications Magazine, June 2002.

[5] IEEE 802.16j PAR. Amendment to IEEE Standard for Local and Metropolitan Area Networks - Part 16: Air Interface for Fixed and Mobile Broadband Wireless Access Systesm - Multihop Relay Specification. IEEE, Mar. 2006.

[6] T. Irnic, D. C. Schultz, R. Pabst, and P. Wienert. Capacity of a Relaying Infrastructure for Broadband Radio Coverage of Urban Areas. IEEE 58th VTC 2003-Fall

[7] K. Park, C. G. Kang, D. Chang, S. Song, J. Ahn, and J. Ihm. Relayenhanced Cellular Performance of OFDMATDD System for Mobile Wireless Broadband Services. ICCCN 2007, In Proc. of 16th, 13-16 Aug. 2007.

[8] O. Oyman. OFDM2A: A Centralized Resource Allocation Policy for Cellular Multi-hop Networks. $40^{\text {th }}$ Asilomar Conference on Signals, Systems and Computers, pages 656 - 660.

[9] M. Kaneko and P. Popovski. Adaptive Resource Allocation in Cellular OFDMA System with Multiple Relay Stations. Proc. of IEEE 65th VTC 2007-Spring, pages $3026-3030$.

[10] T. Girici, C. Zhu, J. Agre, and A. Ephremides. Proportional Fair Scheduling Algorithm in OFDMA-based Wireless Systems with QoS Constraints. 12th International OFDM Workshop (Inowo 07), Hamburg, Germany, 2007.

[11] IEEE 802.16j Relay Task Group. Channel Models and Performance Metrics for IEEE 802.16j Relay Task Group. IEEE, 2006-05-01.

[12] IEEE 802.16 Broadband Wireless Access Working Group. Multihop System Evaluation Methodology: Traffic Models. IEEE, 2006-05-01. 